\title{
Enhancement of Low Quality Fingerprints Based on Anisotropic Filtering*
}

\author{
Xinjian Chen, Jie Tian ${ }^{* *}$, Yangyang Zhang, and Xin Yang \\ Center for Biometrics and Security Research, \\ Key Laboratory of Complex Systems and Intelligence Science, Institute of Automation, \\ Chinese Academy of Sciences, Graduate School of the Chinese Academy of Science, \\ P.O. Box 2728, Beijing 100080, China \\ tian@doctor.com, jie.tian@mail.ia.ac.cn \\ http: //www. fingerpass.net
}

\begin{abstract}
The enhancement of the low quality fingerprint is a difficult and challenge task. This paper proposes an efficient algorithm based on anisotropic filtering to enhance the low quality fingerprint. In our algorithm, an orientation filed estimation with feedback method was proposed to compute the accurate fingerprint orientation. The gradient-based approach was firstly used to compute the coarse orientation. Then the reliability of orientation was computed from the gradient image. If the reliability of the estimated orientation is less than pre-specified threshold, the orientation will be corrected by the mixed orientation model. And an anisotropic filtering was used to enhance the fingerprint, with the advantages of its efficient ridge enhancement and its robustness against noise in the fingerprint image. The proposed algorithm has been evaluated on the databases of Fingerprint verification competition (FVC2004). Experimental results confirm that the proposed algorithm is effective and robust for the enhancement of the low quality fingerprint.
\end{abstract}

\section{Introduction}

There are still many challenging tasks in fingerprint recognition. One of them is the enhancement of low quality fingerprints. The effect of enhancement of poor quality fingerprints is seriously affects the performance of the whole recognition system.

Many image enhancement techniques have been developed for poor quality images. Shi et al[1] proposed a new feature Eccentric Moment to locate the blurry boundary using the new block feature of clarified image for segmentation. Zhou et.al [2] proposed a model-based algorithm which is more accurate and robust to dispose the degraded fingerprints. Lin et al [3] made use of Gabor filter banks to enhance

* This paper is supported by the Project of National Science Fund for Distinguished Young Scholars of China under Grant No. 60225008, the Key Project of National Natural Science Foundation of China under Grant No. 60332010, the Project for Young Scientists' Fund of National Natural Science Foundation of China under Grant No.60303022, and the Project of Natural Science Foundation of Beijing under Grant No.4052026.

* Corresponding author. Tel: 8610-62532105; Fax: 8610-62527995, Senior Member, IEEE.

D. Zhang and A.K. Jain (Eds.): ICB 2006, LNCS 3832, pp. $302-308,2005$.

(C) Springer-Verlag Berlin Heidelberg 2005 
fingerprint images and reported to achieve good performance. Yang et al.[4] proposed a modified Gabor filter to enhance fingerprints, specified parameters deliberately through some principles instead of experience, preserved fingerprint image structure and achieved image enhancement consistency. Willis et al [5] proposed a Fourier domain based method that boosts up a low quality fingerprint image by multiplying the frequency spectrum by its magnitude.

This paper proposes an efficient algorithm based on anisotropic filtering to enhance the low quality fingerprint. The main steps of the algorithm include: normalization, orientation field estimation, reliability of orientation computing, orientation correction, region mask estimation and filtering. In our algorithm, an orientation filed estimation with feedback method was proposed to compute the accurate fingerprint orientation, and an anisotropic filtering was used to enhance the fingerprint

This paper is organized as follows. Section 2 indicates out the details of the enhancement of fingerprint images. Section 3 shows the performance of the proposed algorithm by experiments. Section 4 gives out our conclusion.

\section{Fingerprint Enhancement Algorithm}

The flowchart of the proposed fingerprint enhancement algorithm is shown in figure 1 .

\subsection{Normalization}

Normalization is performed to decrease the dynamic range of the gray scale between ridges and valleys of the image, which facilitates the subsequent enhancement steps. In this paper, Lin et al [3]'s method has been used to process the normalization. The image intensity values were standardized by adjusting the range of grey-level values so that it lies within a desired range of values.

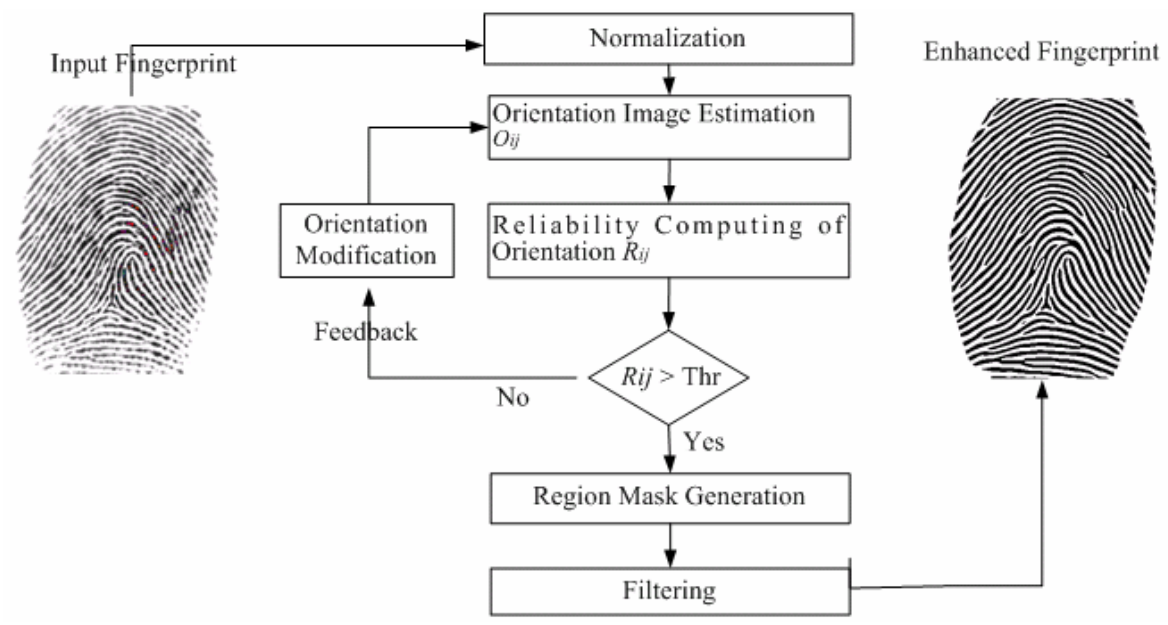

Fig. 1. The flowchart of the proposed enhancement algorithm 


\subsection{Orientation Field Estimation with Feedback}

We proposed an orientation field estimation with feedback method to get the accurate fingerprint orientation. First, the gradient-based approach was used to compute the coarse orientation. Then we compute the reliability of orientation from the gradient image. If the reliability of the estimated orientation rij is less than threshold $t h r$, the orientation will be corrected by the proposed mixed orientation model, otherwise the estimated orientation was taken as the true orientation.

\subsubsection{The Gradient-Based Approach}

In our algorithm, the gradient-based approach proposed by Lin et al [3] was used to compute the coarse orientation. But in our algorithm we divide the normalized image into an odd block of size $\left(15^{*} 15\right)$ instead of $\left(16^{*} 16\right)$.

\subsubsection{Reliability of Orientation Computing}

An additional value rij is associated with each orientation element Oij to denote the reliability of the orientation. The value rij is low for noise and seriously corrupted regions and high for good quality regions in the fingerprint image. The reliability rij is derived by the coherence of the gradient Gij within its neighborhood. It is defined as follows:

$$
r_{i j}=\frac{\left|\sum_{W}\left(G_{i, x}, G_{j, y}\right)\right|}{\sum_{W}\left|\left(G_{i, x}, G_{j, y}\right)\right|}=\frac{\sqrt{\left(G_{x x}-G_{y y}\right)^{2}+4 G_{x y}^{2}}}{G_{x x}+G_{y y}}
$$

Where $\left(G_{i, x}, G_{j, y}\right)$ is the squared gradient, $G_{x x}=\sum_{w} G_{x}{ }^{2}, G_{y y}=\sum_{w} G_{y}{ }^{2}$, $G_{x y}=\sum_{w} G_{x} \cdot G_{y}$ and $\left(G_{x}, G_{y}\right)$ is the local gradient. W is taken as $11 * 11$ block around (i,j).

\subsubsection{Orientation Correction}

The mixed orientation model is consisted of two parts, polynomial model and singular model. Due to the smoothness of the original orientation field, we could choose proper polynomial curves to approach it. We map the orientation field to a continuous complex plane [2]. Denote $\theta(x, y)$ as the orientation field. The mapping is defined as:

$$
\mathrm{U}=\mathrm{R}+\mathrm{iI}=\cos (2 \theta)+\mathrm{i} \sin (2 \theta)
$$

where $\mathrm{R}$ and $\mathrm{I}$ denote the real part and image part of the unit-length complex respectively.

To globally approach the function R and I, a common bivariate polynomial model is chosen for them respectively, which can be formulated as: 


$$
\left(\begin{array}{lll}
1 & \cdots & x^{n}
\end{array}\right) \cdot\left[\begin{array}{cccc}
p_{00} & p_{01} & \cdots & p_{0 n} \\
p_{10} & p_{11} & \cdots & p_{1 n} \\
\vdots & \vdots & \ddots & \vdots \\
p_{n 0} & p_{n 1} & \cdots & p_{n n}
\end{array}\right] \cdot\left(\begin{array}{c}
1 \\
y \\
\vdots \\
y^{n}
\end{array}\right)
$$

where the order $\mathrm{n}$ can be determined ahead.

It is difficult to be modeled with polynomial functions near the singular points. The orientation model proposed by Sherlock and Monro [6] is added at each singular point, and we name it as the singular model. The model allows a consistent directional map to be calculated from the position of the cores and deltas only. In this model the image is located in the complex plane and the orientation is the phase of the square root of a complex rational function with the fingerprint macro-singularities Let $c_{i}\left(i=1 . . n_{c}\right)$ and $d_{i}\left(i=1 . . n_{d}\right)$ be the coordinates of the cores and deltas respectively; the orientation $O^{\prime}$ at each point $(\mathrm{x}, \mathrm{y})$ is calculated as:

$$
O^{\prime}(z)=O_{0}+\frac{1}{2}\left[\sum_{i=1}^{n_{d}} \arg \left(z-d_{i}\right)-\sum_{i=1}^{n_{c}} \arg \left(z-c_{i}\right)\right]
$$

where $O_{0}$ is the background orientation (we set $O_{0}=0$ ), and the function $\arg (\mathrm{z}$ ) returns the argument of the complex number $\mathrm{z}(\mathrm{x}, \mathrm{y})$.

To combine the polynomial model with singular model smoothly, a weight function is defined for singular model, its weight at $(\mathrm{x}, \mathrm{y})$ is defined as:

$$
\begin{gathered}
w= \begin{cases}0 & \text { if }\left(\sum_{i=1}^{k} w_{i}>1\right) \\
1-\sum_{i=1}^{k} w_{i} & \text { otherwise }\end{cases} \\
w_{i}= \begin{cases}0 & \text { if }\left(D_{i}(x, y)>r_{i}\right) \\
1-D_{i}(x, y) / r_{i} & \text { otherwise }\end{cases}
\end{gathered}
$$

where $\mathrm{k}$ is the number of singular points, $\mathrm{i}$ is the ordinal number of singular points, $D_{i}(x, y)$ is the distance between point $(\mathrm{x}, \mathrm{y})$ and i-th singular point, $r_{i}$ is i-th singular point's effective radius.

Finally, the mixed model for the whole fingerprint's orientation field can be formulated as:

$$
O_{m}=(1-w) \cdot \theta+w \cdot O^{\prime}
$$

In order to implement the orientation correction algorithm, the position and type of singular points are need to detected. In our algorithm, the Poincare index method is 
used to detect the singular points. On the other hand, many parameters need to be ascertained. Some of them are initiated and modified based on the experiments while others are computed by least square method.

\subsection{Region Mask Generation}

In this step, we will classify each pixel in an input fingerprint image into a recoverable region or an unrecoverable region. In our algorithm, an optimal linear classifier has been trained for the classification per block and the criteria of minimal number of misclassified samples are used. Morphology has been applied as post-processing to reduce the number of classification errors. The detailed algorithm can be seen from our previous work [7].

\subsection{Fingerprint Filtering}

In the proposed algorithm we replaced the Gabor filter[3] with an anisotropic one, which was proved to be robust and efficient for the filtering of the fingerprint ridges. The structure adaptive anisotropic filtering [8] was modified for fingerprint image filtering. We use both a local intensity orientation and an anisotropic measure to control the shape of the filter. The filter kernel applied to fingerprint image at each point $(\mathrm{x}, \mathrm{y})$ is defined as follows:

$$
\begin{gathered}
h(x, y, \psi)=c_{1}+c_{2} \cdot \exp \left(-\frac{x_{\psi}^{2}}{2 \sigma_{1}^{2}}-\frac{y_{\psi}^{2}}{2 \sigma_{2}^{2}}\right) \cdot \frac{\sin f \cdot x_{\psi}}{f \cdot x_{\psi}} \\
x_{\psi}=x \cos \psi+y \sin \psi \\
y_{\psi}=-x \sin \psi+y \cos \psi
\end{gathered}
$$

$\mathrm{c} 1, \mathrm{c} 2, \sigma_{1}^{2}, \sigma_{2}^{2}$ are empirical parameters,c1=-1, c2=2, $\sigma_{1}^{2}=4, \sigma_{2}^{2}=2$ in our algorithm. $\mathrm{f}$ is a parameter related to the ridge frequency. Applying a 2D Fourier transform to Equation (8), we obtain the filter's frequency response:

$$
\begin{gathered}
H(u, v, \psi)=c_{1} \cdot 4 \pi^{2} \delta(u, v)+2 \pi \cdot c_{2} \sigma_{1} \sigma_{2} \cdot \exp \left(-\frac{u_{\psi}^{2}}{2 \sigma_{u}^{2}}-\frac{v_{\psi}^{2}}{2 \sigma_{v}^{2}}\right) * G\left(u_{\psi}\right) \\
G\left(u_{\psi}\right)=\left\{\begin{array}{rr}
\frac{1}{2 f} & \left|u_{\psi}\right|<2 \pi f \\
0 & \text { otherwise }
\end{array}\right. \\
u_{\psi}=u \cos \psi+v \sin \psi
\end{gathered}
$$




$$
v_{\psi}=-u \sin \psi+v \cos \psi
$$

Where * stands for convolution, $\sigma_{u}=1 / 2 \pi \sigma_{1}, \sigma_{v}=1 / 2 \pi \sigma_{2}$

Let $\mathrm{G}$ be the normalized fingerprint images, $\mathrm{O}$ be the orientation image and $\mathrm{R}$ be the recoverable mask, the enhanced image $F(i, j)$ is obtained as follows:

$$
F(i, j)=\left\{\begin{array}{lr}
255 & \text { if } R(i, j)=0 \\
\sum_{u=-w_{f} / 2}^{w_{f} / 2} \sum_{v=-w_{f} / 2}^{w_{f} / 2} h(u, v: O(i, j)) \cdot G(i-u, j-v) & \text { otherwise }
\end{array}\right.
$$

where $w_{f}=13$ specifies the size of the filters.

\section{Experimental Results}

The proposed algorithm has been evaluated on the databases of FVC2004 [9]. As the limits of pages, only the results on FVC2004 DB2 were listed in this paper.

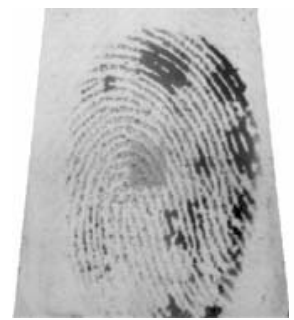

(a)

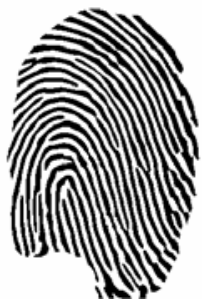

(b)

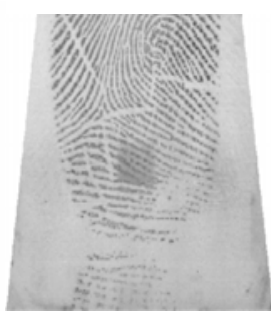

(c)

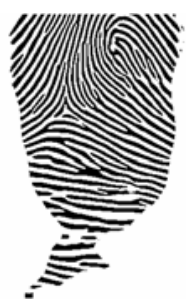

(d)

Fig. 2. Some examples of low quality fingerprints and their enhanced results in FVC2004 DB2. (a) Original image, very dry, (b) Enhanced image of (a), (c) Original image, with scars, (d) Enhanced image of (c).

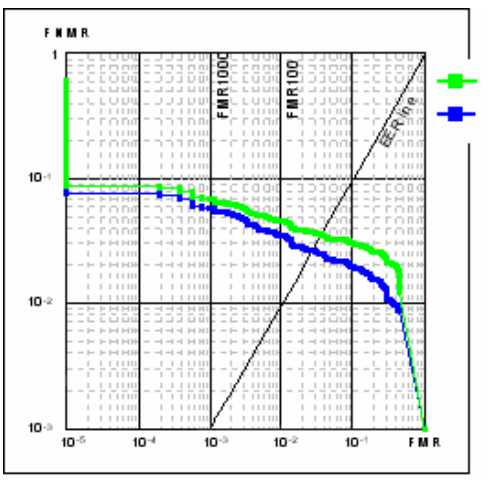

without feedback with feedback

Fig. 3. The comparison of the algorithm with and without feedback method on FVC2004 DB2 
Figure 2 show some examples of low quality fingerprints and their enhanced results in FVC2004 DB2. It can be seen form figure that these poor fingerprints (very dry, with many scars) are enhanced well. The average time for enhancing a fingerprint is about 0.32 second on PC AMD Athlon $1600+(1.41 \mathrm{GHz})$.

Experiments were also done to compare the orientation estimation algorithm with and without feedback method. The comparison results on FVC2004 DB2 are shown in figure 3. The EER was 2.59 for the algorithm with feedback method, while 3.49 for the algorithm without feedback method. It is clear that the performance the recognition algorithm was improved by the feedback method.

\section{Conclusion}

In this paper, an orientation filed estimation with feedback method was proposed to compute the accurate fingerprint orientation. And an anisotropic filtering was applied to enhance the fingerprint, with the advantages of the efficient ridge enhancement and robustness against noise in the fingerprint image. Experimental results confirm that our algorithm is effective and robust for the enhancement of the low quality fingerprint.

\section{References}

1. C. Shi, Y.C. Wang, J. Qi, K. Xu, A New Segmentation Algorithm for Low Quality Fingerprint Image, ICIG 2004, pp.314-317.

2. J. Zhou and J. W. Gu, A Model-based Method for the computation of Fingerprints' Orientation Field, IEEE Trans. On Image Processing, Vol. 13, No. 6, pp.821-835, 2004

3. L. Hong, Y. Wan, A. K Jain, Fingerprint Image Enhancement: Algorithm and Performance Evaluation. IEEE Trans. PAMI, 20(8), pp.777-789, 1998.

4. J. W. Yang, L. F. Liu, T. Z. Jiang, Y. Fan, A modified Gabor filter design method for fingerprint image enhancement, Pattern Recognition, Vol.24, pp.1805-1817, 2003.

5. A.J. Willis, L. Myers, A Cost-effective Fingerprint Recognition System for Use with Lowquality Prints and Damaged Fingertips. Pattern Recognition, 34(2), pp.255-270, 2001

6. B. Sherlock and D. Monro, A Model for Interpreting Fingerprint Topology, Pattern Recognition, v. 26, no. 7, pp. 1047-1095, 1993.

7. X. J. Chen, J. Tian, J. G. Cheng, X. Yang, Segmentation of Fingerprint Images Using Linear Classifier. EURASIP Journal on Applied Signal Processing, Vol. 2004, No. 4, pp.480-494, Apr.2004

8. G.Z. Yang, P. Burger, D.N. Firmin and S.R. Underwood, Structure Adaptive Anisotropic Filtering. Image and Vision Computing 14: 135-145, 1996.

9. Biometric Systems Lab, Pattern Recognition and Image Processing Laboratory, Biometric Test Center, http://bias.csr.unibo.it/fvc2004/. 